\title{
Leptin, IL-6, and suPAR reflect distinct inflammatory changes associated with adiposity, lipodystrophy and low muscle mass in HIV-infected patients and controls
}

\author{
Anne Langkilde ${ }^{1 *}$, Janne Petersen ${ }^{1}$, Jens Henrik Henriksen², Frank Krieger Jensen³ ${ }^{3}$ Jan Gerstoft ${ }^{4}$,
} Jesper Eugen-Olsen ${ }^{1}$ and Ove Andersen ${ }^{1,5}$

\begin{abstract}
Background: HIV-infected patients could exhibit accelerated ageing, since age-associated complications like sarcopenia; increased inflammation; lipodystrophy with loss of subcutaneous adipose tissue and/or gain of visceral adipose tissue (VAT); and cardiovascular disease occur at an earlier age. Inflammation is involved in age-associated complications. However, it is not understood whether it is the same inflammatory changes that are involved in the various ageing-associated complications. Our objective was to study whether leptin, interleukin 6 (IL-6), and soluble urokinase plasminogen activator receptor (suPAR) were associated distinctively with adiposity, lipodystrophy and sarcopenia, in HIV-infected patients and healthy Controls.

Results: Systemic leptin levels were significantly higher in patients with lipodystrophy than without, whereas there was no difference in IL-6 or suPAR levels. Leptin was significantly positively associated with fat mass index (FMI) and abdominal VAT, but not with lean mass index (LMI). IL-6 was significantly associated with both FMI and VAT, and low LMI. High suPAR was associated with low LMI, and weakly with high FMI and VAT.

Conclusions: Leptin reflected adiposity- and lipodystrophy-related inflammation, but not sarcopenia. IL-6 reflected both adiposity-, but also sarcopenia-related inflammation; and suPAR was a marker of sarcopenia-related inflammation. Our results indicate that different inflammatory processes can be active simultaneously contributing to the systemic low grade inflammatory state. Identifying major contributors to circulating leptin, IL-6, and suPAR levels could levels could therefore improve our understanding of which inflammatory processes are involved in the various age-related complications.
\end{abstract}

Keywords: Inflammation, Aging, Sarcopenia, HIV, suPAR, Obesity, Lipodystrophy, IL-6, Leptin

\section{Background}

Inflammation could be a driver of biological ageing, since inflammation increases with age; is elevated in ageing-associated conditions like sarcopenia, and dysmetabolism; and is a risk factor for cardiovascular disease, type 2 diabetes, cancer, and overall mortality [1-6]. Accordingly, HIV-infected patients could exhibit accelerated ageing, as combination antiretroviral

\footnotetext{
* Correspondence: annelangkilde@gmail.com

${ }^{1}$ Clinical Research Centre, Copenhagen University Hospital, Hvidovre, Kettegård Alle 30, DK-2650 Hvidovre, Denmark

Full list of author information is available at the end of the article
}

therapy (cART)-treated HIV-infected patients exhibit higher inflammatory levels and develop cardiovascular disease, type 2 diabetes, and maybe sarcopenia at a younger age than population controls [7-11]. Moreover, $30-60 \%$ of HIV-infected patients exhibit altered adipose tissue (AT) distribution termed lipodystrophy. Lipodystrophy is characterised by loss of SAT (lipoatrophy) and/or gain of visceral adipose tissue (VAT) (lipohypertrophy), resembling an extreme form of ageassociated AT redistribution. Lipodystrophy is caused by a combination of the infection and cART [12-16], and 
is associated with insulin resistance, systemic and adipose tissue inflammation $[17,18]$.

It is well-established that inflammation is elevated in the majority of age-associated complications. However, it is still not understood whether this is a general inflammatory mechanism, or whether the inflammatory biomarkers reflect different co-existing pathophysiological processes. A major limitation for understanding this, has been inconsistent adjustment for confounders with major impact on inflammation, such as not assessing muscle mass when assessing fat mass. Our aim was therefore to study whether leptin, interleukin 6 (IL-6), and soluble urokinase plasminogen activator receptor (suPAR) were associated distinctively with adiposity, lipodystrophy and sarcopenia, in HIV-infected patients and healthy Controls.

Leptin is a central adipokine produced in levels proportional to fat cell mass $[19,20]$. Leptin integrates inflammation, metabolism and neuroendocrine signalling thereby regulating energy consumption and storage. Obesity is associated with increased leptin levels, and leptin resistance may be a characteristic of obesity contributing to insulin resistance and lipotoxicity [20]. Leptin levels are altered in lipodystrophy, and leptin therapy has been applied to improve insulin sensitivity in HIV-associated lipoatrophy, but with diverging results [21-23]. It is not established whether leptin levels are altered in lipodystrophy merely as a result of the AT mass, or whether AT redistribution affects leptin levels.

IL-6 is a pleiotropic cytokine produced by numerous cell types such as adipocytes, myocytes, and leukocytes. Increased IL-6 levels are associated with obesity, muscle catabolism, cardiovascular disease, and mortality [1, 24-26]. Paradoxically, IL-6 is also produced by skeletal muscle during exercise where it mediates beneficial effects of exercise, independently of muscle damage [25]. IL-6 is elevated in HIV, but IL-6 levels appear not to be affected in lipodystrophy $[8,18]$. How IL-6 reflects and affects AT and muscle mass in HIV-infection and lipodystrophy is not clear.

suPAR is produced when UPAR is cleaved from the cell surface, primarily from activated $\mathrm{T}$ cells, monocytes, neutrophils and endothelial cells. suPAR correlates with other inflammatory proteins such as TNF- $\alpha$, and suPAR levels are elevated in a variety of diseases like HIV, sepsis, cardiovascular disease, and cancer. The function of suPAR is not well-established, but patients with the highest suPAR levels generally have the worst prognosis $[2,27,28]$. In contrast to many other inflammatory markers, suPAR associates weakly with AT measures, but strongly with low lean mass $[2,29,30]$. suPAR could therefore reflect a distinct type of inflammation related to sarcopenia but not AT.

We found that leptin reflected adiposity- and lipodystrophy-related inflammation, but not sarcopenia. IL-6 reflected both adiposity-, but also sarcopenia-related inflammation; and suPAR was a marker of sarcopeniarelated inflammation. Our results indicate that different inflammatory processes can be active simultaneously contributing to the systemic low grade inflammatory state.

\section{Results}

We included 60 HIV-infected patients, 24 with lipodystrophy (Lipo), 36 without lipodystrophy (Non-Lipo), and 16 healthy Controls. One Control was excluded from data analyses due to recent hip-surgery, see Fig. 1. Eight patients did not have DXA scans made. Two patients did not have CT scans made.

\section{Adipose tissue distribution and metabolic parameters in lipodystrophy subgroups}

The Lipo group comprised: 10 patients with lipoatrophy (Atrophy), 4 patients with lipohypertrophy (Hyper), and 10 patients with both lipoatrophy and lipohypertrophy (Mix), see Fig. 1. Mean SAT was $101 \mathrm{~cm}^{2}$ for Atrophy, $300 \mathrm{~cm}^{2}$

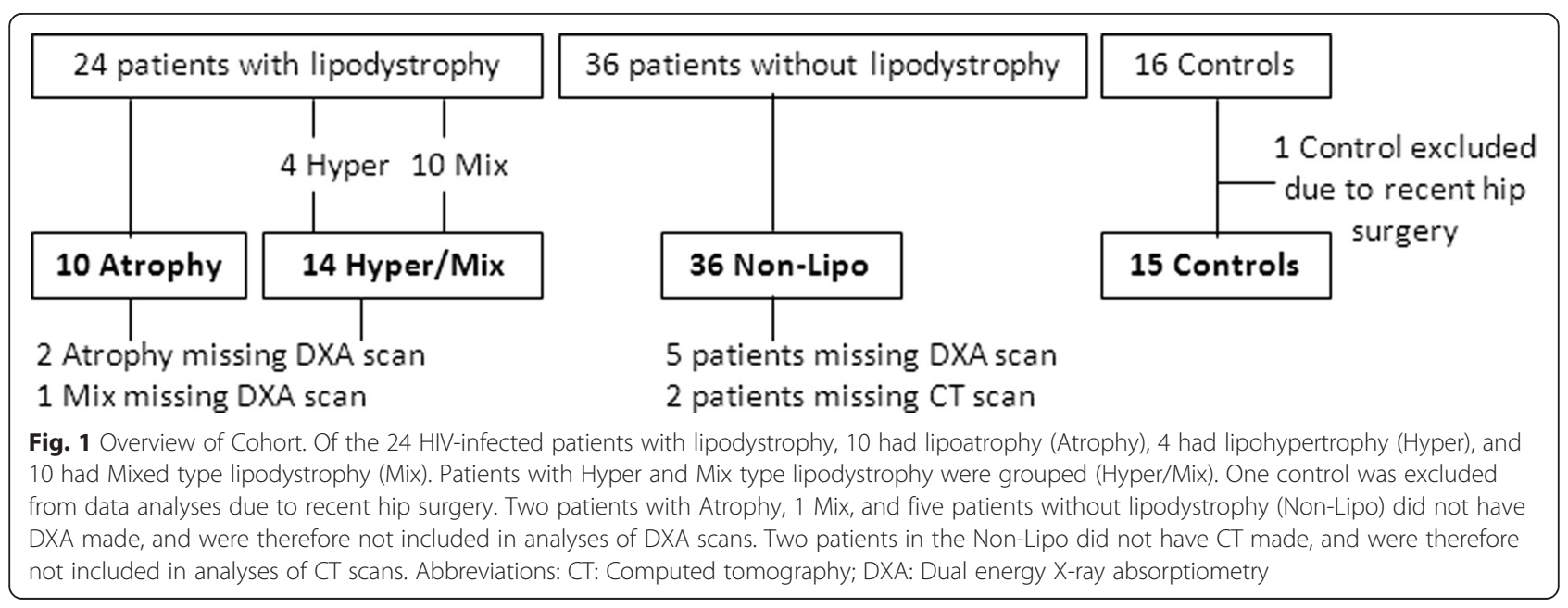


for Hyper, and $221 \mathrm{~cm}^{2}$ for Mix. Mean VAT was $128 \mathrm{~cm}^{2}$ for Atrophy, $285 \mathrm{~cm}^{2}$ for Hyper, and $222 \mathrm{~cm}^{2}$ for Mix. The mean VAT/SAT ratio was 1.5 for Atrophy, 1.0 for Hyper, and 1.2 for Mix. Mean homeostatic model assessment of insulin resistance (HOMA-IR) was 0.9 for Atrophy; 2.1 for Hyper; and 4.9 for Mix. Metabolic syndrome was present in 10 \% in Atrophy; 75 \% in Hyper; and 90 \% in Mix. Since lipoatrophy and lipohypertrophy could be caused by distinct disease processes, we decided to analyse Lipo in subgroups. Hyper and Mix (Hyper/Mix) were grouped for further analyses, due to the low number of patients with lipohypertrophy, and the fact that Hyper and Mix had similar VAT and metabolic risk factors compared to Atrophy.

\section{Baseline characteristics of atrophy, hyper/mix, non-lipo and controls}

Baseline characteristics for Atrophy, Hyper/Mix, NonLipo and Controls are shown in Table 1. Patients in the Hyper/Mix group were significantly older than NonLipo. Both Atrophy and Hyper/Mix had significantly longer HIV and treatment duration, and a higher percent of patients previously treated with thymidine nucleoside reverse transcriptase inhibitors (tNRTI) than the NonLipo group. Smoking was significantly more prevalent, and triglyceride and very low density lipoprotein (VLDL) levels were significantly higher in Non-Lipo compared to Controls. BMI, FMI, SAT and $t$ LMI was significantly

Table 1 Baseline Characteristics

\begin{tabular}{|c|c|c|c|c|}
\hline Variable & Atrophy $(\mathrm{N}=10)$ & Hyper/Mix $(\mathrm{N}=4+10)$ & Non-Lipo $(N=36)$ & Controls $(N=15)$ \\
\hline \multicolumn{5}{|l|}{ Demography and lifestyle } \\
\hline Age (years) & $50.1(46.2 ; 47.5)$ & $58.1(54.0 ; 64.1)^{*}$ & $48.3(40.1 ; 53.9)$ & $47.6(40.9 ; 62.3)$ \\
\hline Smoking (N (\%)) & $4(40.0 \%)$ & $2(14.3 \%)$ & $12(33.3 \%)$ & $1(6.7 \%)^{*}$ \\
\hline \multicolumn{5}{|l|}{ Body composition } \\
\hline BMI $\left(\mathrm{kg} / \mathrm{m}^{2}\right)$ & $21.1(19.4 ; 24.1)^{*}$ & $27.2(24.7 ; 29.3)$ & $25.5(23.2 ; 28.2)$ & $25.4(22.2 ; 29.7)$ \\
\hline FMI $\left(\mathrm{kg} / \mathrm{m}^{2}\right)$ & $2.4(1.6 ; 3.2)^{*}$ & $5.4(4.0 ; 7.1)$ & $4.8(3.6 ; 6.7)$ & $4.8(4.4 ; 7.5)$ \\
\hline $\operatorname{VAT}\left(\mathrm{cm}^{2}\right)$ & $136.7(91.7 ; 160.4)$ & $251.0(178.9 ; 280.9)^{*}$ & $136.4(96.8 ; 194.3)$ & $120.2(93.5 ; 159.8)$ \\
\hline SAT $\left(\mathrm{cm}^{2}\right)$ & $105.5(56.4 ; 129.1)^{*}$ & $254.8(203.2 ; 294.6)$ & $197.4(152.1 ; 264.1)$ & $253.5(146.2 ; 310.9)$ \\
\hline VAT/SAT & $1.4(1.1 ; 1.8)^{*}$ & $0.8(0.7 ; 1.6)^{*}$ & $0.7(0.5 ; 0.9)$ & $0.5(0.4 ; 0.8)$ \\
\hline tLMI $\left(\mathrm{kg} / \mathrm{m}^{2}\right)$ & $17.1(15.7 ; 18.4)^{*}$ & $20.3(18.4 ; 20.6)$ & $18.4(17.1 ; 20.6)$ & $18.4(18.0 ; 20.6)$ \\
\hline ILMI $\left(\mathrm{kg} / \mathrm{m}^{2}\right)$ & $5.5(5.0 ; 6.6)$ & $6.3(6.0 ; 6.7)$ & $6.1(5.7 ; 6.7)$ & $6.5(6.2 ; 7.0)$ \\
\hline \multicolumn{5}{|l|}{ Metabolism } \\
\hline Triglyceride & $1.3(1.1 ; 2.1)$ & $1.4(1.2 ; 2.4)$ & $1.6(1.0 ; 2.0)$ & $0.9(0.7 ; 1.1)^{*}$ \\
\hline Cholesterol & $5.3(4.6 ; 6.4)$ & $5.5(4.8 ; 6.6)$ & $5.4(4.8 ; 6.4)$ & $5.4(4.7 ; 5.7)$ \\
\hline HDL & $1.4(1.2 ; 1.5)$ & $1.3(1.0 ; 1.5)$ & $1.3(1.1 ; 1.5)$ & $1.4(1.3 ; 1.6)$ \\
\hline VLDL & $0.6(0.5 ; 0.9)$ & $0.5(0.5 ; 0.9)$ & $0.7(0.5 ; 0.9)$ & $0.4(0.3 ; 0.5)^{*}$ \\
\hline LDL & $3.2(2.5 ; 4.2)$ & $3.2(2.7 ; 3.8)$ & $3.4(3.0 ; 4.5)$ & $3.1(2.6 ; 3.8)$ \\
\hline HOMA-IR & $0.9(0.5 ; 1.2)$ & $2.4(1.6 ; 4.1)^{*}$ & $0.9(0.5 ; 2.1)$ & $0.5(0.5 ; 1.3)^{*}$ \\
\hline Metabolic syndrome, N(\%) & 1 (10.0 \%) & $12(85.7 \%)^{*}$ & 14 (38.9\%) & $2(13.3 \%)$ \\
\hline \multicolumn{5}{|l|}{ HIV } \\
\hline HIV duration (years) & $19.5(17.8 ; 25.6)^{*}$ & $20.8(16.5 ; 24.4)^{*}$ & $11.0(4.9 ; 16.2)$ & - \\
\hline CART duration (years) & $15.3(12.6 ; 18.4)^{*}$ & $15.2(13.6 ; 16.3)^{*}$ & $5.8(3.5 ; 13.0)$ & - \\
\hline Current tNRTI & $0(0 \%)$ & $0(0 \%)$ & $1(2.8 \%)$ & - \\
\hline Previous tNRTI & $9(100.0 \%)^{* a}$ & $14(100.0 \%)^{*}$ & $18(53.0 \%)^{\mathrm{a}}$ & - \\
\hline HIV RNA $\leq 20$ copies $/ \mathrm{mL}$ & $9(90.0 \%)$ & $14(100.0 \%)$ & $32(88.9 \%)$ & - \\
\hline CD4+ T cells/ $\mu \mathrm{L}$ & $716(569 ; 830)$ & $593(534 ; 910)$ & $535(393 ; 736)$ & $739(598 ; 880)^{*}$ \\
\hline 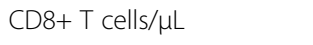 & $1090(802 ; 1160)$ & $654(583 ; 829)$ & $821(597 ;$ 1160) & $397(281 ; 493)^{*}$ \\
\hline CD4+:CD8+ ratio & $0.7(0.6 ; 0.8)$ & $1.0(0.7 ; 1.3)$ & $0.7(0.5 ; 0.9)$ & $1.9(1.5 ; 3.0)^{*}$ \\
\hline
\end{tabular}

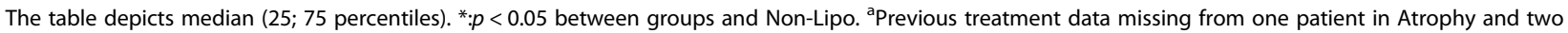
patients in the Non-Lipo group

Abbreviations: BMI body mass index, $C A R T$ combination antiretroviral treatment, FMI fat mass index, HDL high density lipoprotein, HOMA-IR homeostatic model assessment of insulin resistance, IL-6 Interleukin 6, LDL low density lipoprotein, sUPAR soluble urokinase plasminogen activator receptor, SAT subcutaneous adipose tissue, $t N R T I$ thymidine nucleoside reverse transcriptase inhibitors, VAT Visceral adipose tissue, VLDL very low density lipoprotein

We analysed differences between groups and Non-Lipo. Data were analysed using one-way Anova. BMI, FMI, HDL, SAT, triglycerides, VAT/SAT, VLDL, HOMA, and CD4 was analysed using Kruskal-Wallis test. Smoking, metabolic syndrome, previous tNRTI, and HIV RNA $\leq 20$ copies/mL were analysed using Fisher's exact test 
lower in Atrophy compared to Non-Lipo, whereas there was no significant difference between Hyper/Mix and Non-Lipo, and Non-Lipo and Controls. In contrast, VAT was significantly higher in Hyper/Mix compared to Non-Lipo, and both Atrophy and Hyper/Mix had significantly higher VAT/SAT ratio than Non-Lipo. HOMA-IR was significantly higher and metabolic syndrome significantly more prevalent in Hyper/Mix than Non-Lipo, whereas there was no difference between Atrophy and Non-Lipo.

\section{Associations between leptin, IL-6, and suPAR with age,} smoking, lipodystrophy, and HIV

In univariate regression analyses, neither leptin nor IL-6 were associated with higher age, but suPAR tended to be $(p=0.05)$. IL-6 $(p=0.004)$ and suPAR $(p<0.001)$ were significantly higher in daily smokers than non-daily smokers, whereas leptin levels tended to be lower in daily smokers $(39.5 \%$ lower, $p=0.06)$. In univariate regression analyses, leptin levels were significantly lower in Atrophy than Non-Lipo (60\% lower, $p=0.007$ ), and significantly higher in Hyper/Mix than Non-Lipo (105\% higher, $p=0.02$ ), see Fig. 2 and Table 2. In contrast, there was no significant difference in IL-6 or suPAR levels between Lipo subgroups and NonLipo. Leptin levels were not significantly different in Non-Lipo and Controls, whereas IL-6 and suPAR were significantly higher in Non-Lipo compared to Controls (IL-6: $p=0.03$; suPAR: $p=0.02$ ), see Fig. 2 and Table 2. Furthermore, we found no associations between the biomarkers and previous treatment with tNRTI or duration of the infection or cART (data not shown).

\section{Univariate associations between leptin, IL-6, and suPAR} with body composition

Leptin and IL-6 were strongly associated with high FMI and VAT, whereas suPAR was not (Table 2). Leptin was significantly correlated with high $t$ LMI and $l$ LMI; whereas suPAR was significantly correlated with low $t$ LMI and $l$ LMI (Table 2). IL-6 was not significantly correlated with either $t \mathrm{LMI}$ or $l \mathrm{LMI}$.

Interaction analyses for the univariate association between biomarkers and body composition in the four groups

The association between leptin and body composition was significantly modified by group, whereas the association of IL- 6 and suPAR with body composition was not, see Fig. 3. The association between leptin and FMI was stronger for Controls $(p<0.001)$ and Atrophy $(p=0.05)$ than for Non-Lipo, though only significantly different for Controls. Moreover, we found a tendency of leptin to be more strongly associated with VAT for Controls $(p=0.06)$ than for Non-Lipo. The positive association between leptin and $l$ LMI was abrogated in the Hyper/Mix group ( $p=0.01)$, see Fig. 3 .

\section{Relation between fat and muscle mass}

We investigated whether fat and muscle were associated, as found in previous studies. High FMI was significantly associated with high $t$ LMI $(p<0.001)$ and $l \mathrm{LMI}(p<0.001)$, see Fig. 4 left. We tested whether the association between fat and muscle mass was modified by group. In the Hyper/ Mix group, FMI appeared not to be associated with $l$ LMI ( $p=0.08$ versus Non-Lipo) or $t$ LMI $(p=0.08$ versus Non-Lipo). However, when removing an influential outlier from the Hyper/Mix group, the association between FMI and $l$ LMI or $t$ LMI was not significantly different in Hyper/Mix and Non-Lipo ( $p=0.42$; $t$ LMI: $p=0.40$ versus Non-Lipo), as seen in Fig. 4 right.

Adjusted analyses for leptin, IL-6, and suPAR and the associations with age, smoking, lipodystrophy, and HIV Adjusted for group, none of the biomarkers were significantly associated with age. IL-6 and suPAR were significantly elevated in daily smokers, when adjusting for age

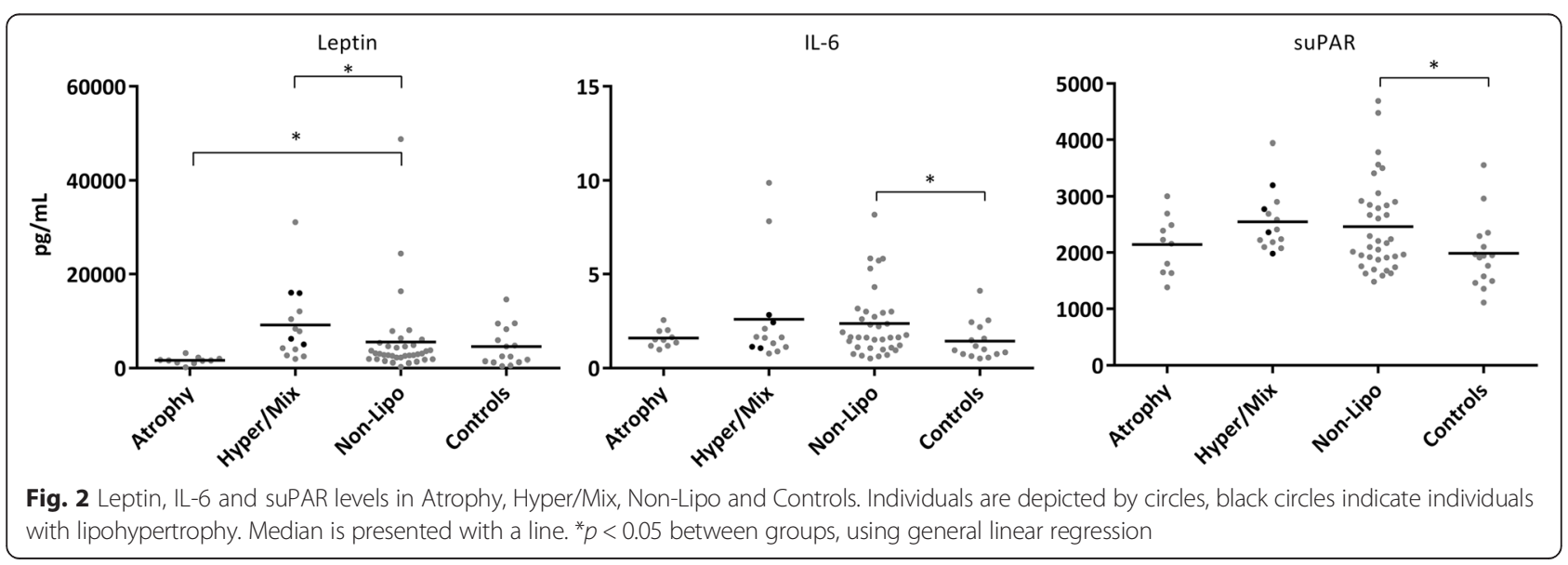


Table 2 Univariate and multiple regression analyses

\begin{tabular}{|c|c|c|c|c|c|c|}
\hline & \multicolumn{3}{|c|}{ Univariate \% Estimate (p) } & \multicolumn{3}{|c|}{ Multiple \% Estimate (p) } \\
\hline & Leptin & IL-6 & SUPAR & Leptin & IL-6 & SUPAR \\
\hline Age (years) & $0.9(0.45)$ & $0.5(0.53)$ & $0.6(0.05)$ & $0.01(1.0)$ & $0.4(0.64)$ & $0.5(0.10)$ \\
\hline Daily smoking & $-39.5(0.06)$ & $65.8(0.004)$ & $33.6(<0.001)$ & $-32.8(0.13)$ & $63.6(0.007)$ & $36.7(<0.001)$ \\
\hline Atrophy vs. Non-Lipo & $-60.0(0.007)$ & $-17.8(0.41)$ & $-11.2(0.24)$ & $-5.1(0.82)$ & $6.2(0.81)$ & $-5.1(0.57)$ \\
\hline Hyper/Mix vs. Non-Lipo & $104.5(0.02)$ & $-0.1(0.99)$ & $6.6(0.47)$ & $128.3(<0.001)$ & $3.3(0.87)$ & $3.4(0.63)$ \\
\hline Non-Lipo vs. Controls & $16.4(0.59)$ & $58.3(0.03)$ & $23.2(0.02)$ & $1.6(0.93)$ & $27.4(0.21)$ & $9.9(0.17)$ \\
\hline $\mathrm{FMI}\left(\mathrm{kg} / \mathrm{m}^{2}\right)$ & $35.3(<0.001)$ & $8.0(0.009)$ & $0.1(0.95)$ & $33.5(<0.001)$ & $15.1(<0.001)$ & $3.5(0.006)$ \\
\hline VAT $\left(\mathrm{cm}^{2}\right)$ & $0.9(<0.001)$ & $0.3(0.001)$ & $0.1(0.19)$ & $0.8(<0.001)$ & $0.5(<0.001)$ & $0.1(0.009)$ \\
\hline$t \mathrm{LMI}\left(\mathrm{kg} / \mathrm{m}^{2}\right)$ & $26.4(<0.001)$ & $-1.3(0.70)$ & $-3.0(0.03)$ & $0.2(0.95)$ & $-10.8(0.003)$ & $-5.5(0.002)$ \\
\hline LMI $\left(\mathrm{kg} / \mathrm{m}^{2}\right)$ & $76.6(<0.001)$ & $-4.9(0.61)$ & $-10.6(0.003)$ & $-0.6(0.95)$ & $-20.7(0.03)$ & $-12.7(<0.001)$ \\
\hline
\end{tabular}

Biomarker levels were transformed using $\log _{2}(\mathrm{x})$ in univariate and multiple analyses. Results are therefore back-transformed using $\left(1-2^{\beta}\right) * 100$ and shown as percent estimates per unit increase. Associations where $p<0.05$ are bold

Abbreviations: BMI body mass index, FMI fat mass index, $t \mathrm{LMI}$ total lean mass index, ILMI leg lean mass index, VAT visceral adipose tissue

Multiple analyses adjustments: Age was adjusted for group. Daily smoking was adjusted for age and group. Group analyses were adjusted for age, smoking, FMI, and ILMI. FMI and VAT analyses were adjusted for age, smoking, group, and /LMI. tLMI and /LMI analyses were adjusted for age, smoking, group, and FMI

and group. We performed multiple regression analyses for the association between biomarkers, body composition and lipodystrophy, adjusted for both FMI and $l$ LMI to account for the interrelation between fat and muscle mass. Results are shown in Table 2. Adjusted for age, smoking, FMI and $l$ LMI we found $128.3 \%$ higher leptin levels in Hyper/Mix compared to Non-Lipo $(p<0.001)$, but no difference between Atrophy or Controls and Non-Lipo. IL-6 and suPAR levels were not altered in Hyper/Mix or Atrophy compared to Non-Lipo, but IL-6
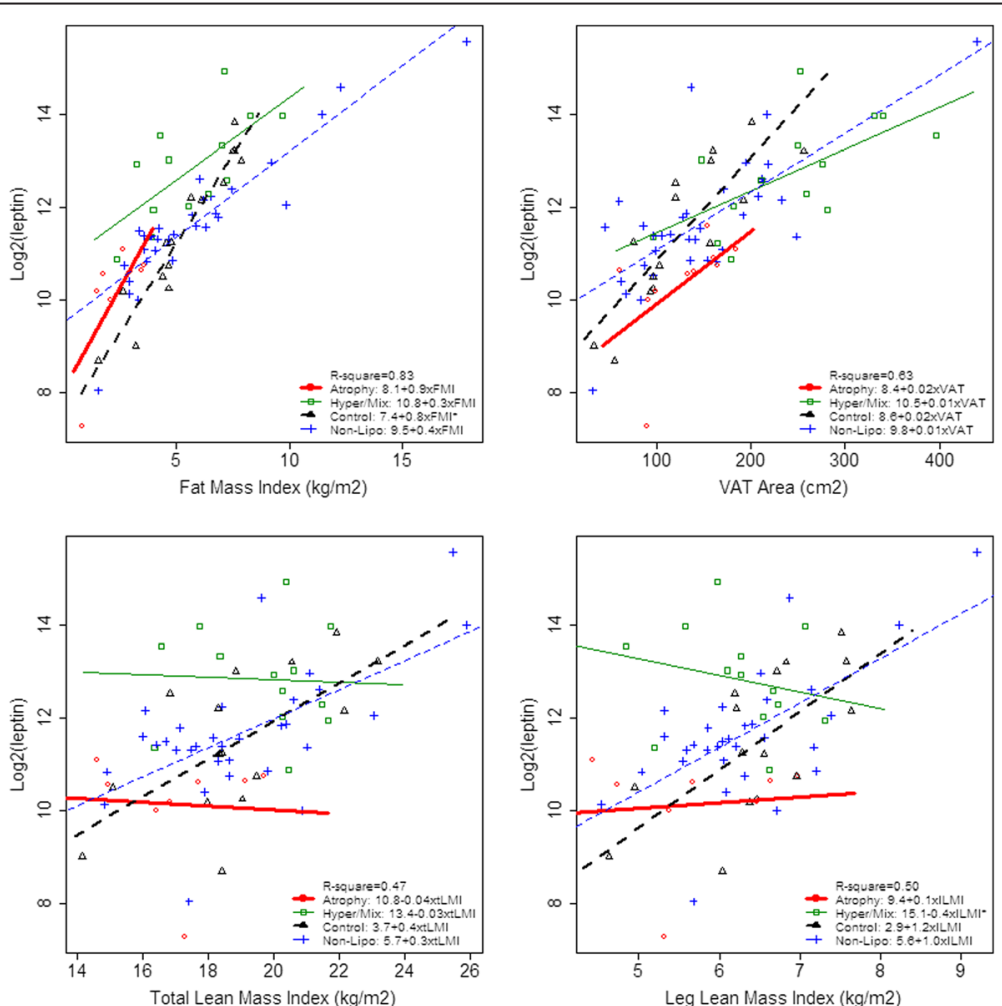

Fig. 3 Leptin levels and Body Composition, stratified by group. The figure shows individuals from each group and group-specific regression lines and the equations for the regression lines. If the association is significantly different from Non-Lipo, the legend is marked with $a^{*}$. Atrophy is presented by red circles and the regression line is thick, solid and red; Hyper/Mix by green and squares, and the regression line is thin, solid and green; Controls by black and triangles, and the regression line is thick, dotted and black. Non-Lipo by blue and plus, and the regression line is thin, dotted, and blue. Abbreviation: VAT: visceral adipose tissue 

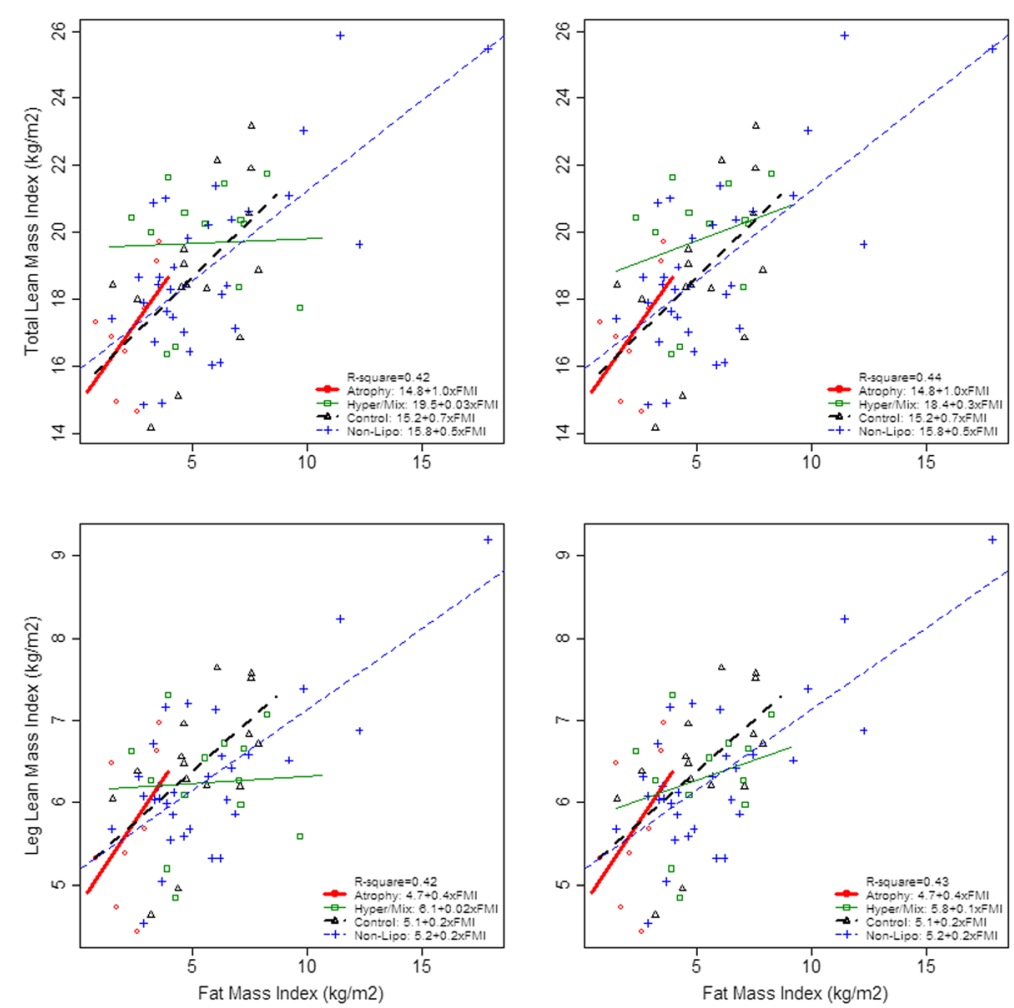

Fig. 4 Association between fat and lean mass index, stratified by group. The figure shows individuals from each group and group-specific regression lines. Atrophy is presented by red circles and the regression line is thick, solid and red; Hyper/Mix by green and squares, and the regression line is thin, solid and green; Controls by black and triangles, and the regression line is thick, dotted and black. Non-Lipo by blue and plus, and the regression line is thin, dotted, and blue. Abbreviation: VAT: visceral adipose tissue

$(p=0.21)$ and suPAR $(p=0.17)$ were higher in Non-Lipo than Controls, though not significantly.

\section{Adjusted analyses for leptin, IL-6, and suPAR and the association with adipose tissue}

When adjusting for age, smoking, group, and $l \mathrm{LMI}$; leptin and IL-6 were strongly associated with FMI and VAT, whereas suPAR was only weakly associated (Table 2). The association between FMI and leptin differed between groups, and was significantly stronger for Atrophy (103.6\% per $\mathrm{kg} / \mathrm{m}^{2}, \mathrm{p}=0.02$ vs. Non-Lipo) and Controls (72.4 \% per $\mathrm{kg} / \mathrm{m}^{2}, p<0.001$ vs. Non-Lipo) than for Non-Lipo (30.0\% per $\mathrm{kg} / \mathrm{m}^{2}$ ). Also the association between FMI and IL-6 (33.0\% per $\mathrm{kg} / \mathrm{m}^{2}, p=0.06$ vs. Non-Lipo) and suPAR ( $9.1 \%$ per $\mathrm{kg} / \mathrm{m}^{2}, p=0.06$ vs. Non-Lipo) tended to be stronger for Controls than for Non-Lipo. We found no difference between the groups for the association between leptin, IL-6, and suPAR with VAT.

\section{Adjusted analyses for leptin, IL-6, and suPAR and the association with lean mass}

When adjusting for age, smoking, group and FMI; IL-6 and suPAR were significantly associated with low $t \mathrm{LMI}$ and $l$ LMI, whereas the positive association between leptin and lean mass was attenuated (Table 2). However, in Controls leptin was still significantly positively associated with both $t$ LMI $\left(16.2 \%\right.$ per $\mathrm{kg} / \mathrm{m}^{2}, p=0.004$ vs. Non-Lipo) and $l$ LMI (59.1 \% per $\mathrm{kg} / \mathrm{m}^{2}, p=0.003$ vs. Non-Lipo).

Exclusion of the influential outlier from the Hyper/ Mix group did not change either the univariate or multiple adjusted analyses significantly. Furthermore, adjusting for HOMA-IR did not change the estimates of the multiple adjusted analyses.

\section{Correlation between biomarkers}

We assessed the association between biomarkers, adjusted for group. Leptin and IL-6 were significantly correlated $(15.5 \%$ per pg/mL IL-6 increase; $p=0.02)$. There was no significant correlation for leptin and suPAR $(-12.1 \%$ per $\mathrm{ng} / \mathrm{mL}$ suPAR increase; $p=0.41)$. IL-6 and suPAR levels correlated significantly (64.3\% per $\mathrm{ng} / \mathrm{mL}$ suPAR increase; $p<0.001)$. The correlations between biomarkers did not differ significantly between groups. 


\section{Discussion}

Inflammation has been intensively studied in age-associated complications. However, it is not clear whether it is the same inflammatory changes that are central in all ageassociated complications. We therefore studied leptin, IL-6 and suPAR that we hypothesised would be associated distinctively with lipodystrophy, adiposity and sarcopenia. Leptin levels were significantly elevated in lipodystrophy of the Hypertrophic or Mixed type (Hyper/Mix) compared to HIV-infected patients without lipodystrophy (Non-Lipo). In contrast, IL-6 or suPAR levels were not changed in lipodystrophy, but tended to be higher in HIV-infected patients than Controls. Leptin was a strong marker of fat mass. In contrast, suPAR showed strong associations with low lean mass, and weak associations with fat mass. IL-6 was a marker of both increased fat and low lean mass. Thus we found leptin, IL- 6 and suPAR to be associated with lipodystrophy, adiposity and sarcopenia distinctively.

For the first time we show that leptin levels were significantly elevated in patients with Hyper/Mix type lipodystrophy compared to Non-Lipo, not only as a result of the fat mass or the insulin sensitivity. In contrast, leptin levels were not significantly lower in patients with lipoatrophy, than what was expected from their low fat mass. Results from other studies suggest that the elevated leptin levels do not appear to be a general feature of age-associated AT re-distribution [19]. The elevated leptin levels in Hyper/ Mix type lipodystrophy could be a physiological response to counteract ectopic fat-mediated lipotoxicity, as leptin exhibits lipotoxicity-protective effects [20]. However, the higher leptin levels could also represent a state of leptin resistance contributing to insulin resistance.

IL-6 and suPAR levels were not altered in lipodystrophy of either subtype, in agreement with most studies [18, 27]. In contrast, IL-6 and suPAR levels were elevated in Non-Lipo compared to Controls, though not significantly in multiple adjusted analyses. Along with the highly elevated CD8+ $\mathrm{T}$ cell counts and the lower CD4+/CD8+ T cell ratio, these findings support that the immune system has not been fully normalised in HIV-infected patients despite successful treatment, in agreement with previous studies [8]. The chronic immune activation could be involved in the earlier onset of comorbidities in HIV-infected patients.

FMI and $t$ LMI and $l$ LMI were positively associated, in agreement with the increase in lean mass required to carry an increased fat mass previously reported [31]. However, in Hyper/Mix the association appeared disturbed, also when removing an influential outlier. Whether this is an artefact, or whether Hyper/Mix type lipodystrophy is associated with dysregulation of muscle homeostasis as in sarcopenic obesity, is not possible to determine in this study.
Leptin and IL-6 were significantly associated with FMI and VAT in both univariate and multiple regression analyses. In contrast, suPAR was only significantly associated with FMI and VAT in multiple regression analyses, in agreement with previous studies [1, 19]. This did not appear to be a result of insulin resistance, since adjusting for HOMA-IR did not change the estimates. When adjusting for fat mass, IL-6 was independently associated with low lean mass, in contrast to the univariate analysis. Moreover, we confirmed our previous finding that suPAR reflects low lean mass in HIV-infected patients and extended this to healthy Controls [29]. Neither the association with suPAR nor IL-6 and low lean mass was explained by self-reported physical activity (data not shown). The association of suPAR with low lean mass may provide new insight into why suPAR is a risk marker for mortality.

Our findings, of inflammatory biomarkers being associated distinctively with age-related processes, are in agreement with other studies, where it was reported that inflammatory biomarkers were distinctively associated with disease severity, and with risk factors leading to disease development [30, 32]. This indicates that different inflammatory processes can be active simultaneously contributing to the systemic low grade inflammatory state. CCAAT-enhancer-binding proteins $(\mathrm{C} / \mathrm{EBP})$ and specificity protein (SP)-1 are transcription factors for both the leptin and IL- 6 genes. Thus, on the molecular level, the association between leptin and IL- 6 and adiposity could be the result of pathways leading to C/EBP and SP-1 binding to the promoter regions of the leptin and IL-6 genes [33-35]. However, the different inflammatory processes also affect each other, as leptin and IL6 affect the production of each other. It is not well understood, whether suPAR is regulated by, or regulates other inflammatory mediators [36, 37].

On the cellular level, adiposity-associated inflammation may result from an increased adipocyte size leading to AT stress. AT stress could induce adipocyte death, inflammation, and macrophage infiltration [38-40]. However, inflammation can also induce AT stress, thereby initiating a vicious cycle. More specifically, enlarged adipocytes may contribute significantly to the elevated leptin levels, since leptin is produced in amounts proportional to fat cell size [41]. AT-derived IL-6 can be produced by stressed adipocytes or by infiltrating macrophages. Elevated IL-6 levels may contribute to disease development, by increasing the expression of cytokines like TNF- $\alpha$ known to induce insulin resistance, or by inducing insulin resistance itself $[5,42,43]$.

Muscle leptin expression is very small, thus leptin does not appear to be involved in muscle pathology [33]. Inflammation could cause muscle atrophy by increasing Nuclear Factor- $\mathrm{kB}$ expression that is known to 
activate atrogen gene expression in muscle [26]. IL-6 may promote muscle catabolism directly through signal transducer and activator of transcription 3 resulting in Atrogin-1 expression. However, IL- 6 anabolic effects have also been reported [24]. IL-6 catabolic effects may first ensue in a persistent inflammatory state and thus be dependent on other inflammatory mediators.

The uPAR ligand urokinase plasminogen activator (uPA) exhibits muscle anabolic properties, independently of its binding to uPAR [44-47]. suPAR may directly affect muscle homeostatis by binding uPA. suPAR is cleaved from the cell surface by proteases involved in extracellular matrix degradation. The increased suPAR levels associated with low muscle mass, could therefore also be a result of the tissue re-modulation taken place during muscle atrophy $[26,28]$. However, underlying pathophysiological processes could give rise to elevated suPAR and IL-6 levels, and low muscle mass, without any direct muscle atrophic effects of suPAR and IL-6.

There are some limitations to our study. The study was cross-sectional, and we could therefore not examine the role of leptin, IL-6, and suPAR in the development of complications. Moreover, our Control and lipodystrophy subgroups were small, and our findings should therefore be confirmed in larger studies. Furthermore, to understand whether the inflammatory changes identified here are indeed part of the pathophysiologic process, studies at the molecular level should be carried out. Our findings for patients with lipoatrophy are representative for relatively normo-metabolic patients, since inclusion criteria were no lipid-lowering or anti-diabetic treatment. Furthermore, our study was designed to assess lipodystrophy, and not lipodystrophy subtypes, and few patients with the lipohypertrophic phenotype were included. We therefore grouped patients with lipohypertrophy and mixed type lipodystrophy. Our findings support those from other studies, in that lipodystrophy phenotypes should be analysed separately [21, 48]. Finally, we could not determine the impact of the HIV infection itself, since no cART naïve HIV-infected group was included.

\section{Conclusion}

Our findings add to the growing knowledge of inflammation involved in adiposity, adipose tissue redistribution and sarcopenia. In line with other studies, we showed that fat mass and lean mass are correlated, which could confound the relation between body composition and inflammation if both muscle and fat is not taken into account. In conclusion, we showed that leptin levels are elevated in Hyper/Mix type lipodystrophy not only as a result of the fat mass. This suggests that the leptin system is central in the pathophysiology of Hyper/Mix type lipodystrophy. In contrast, suPAR appeared to primarily reflect sarcopenia- associated inflammation, whereas IL- 6 was central in both adiposity- and sarcopenia-associated inflammation. These findings suggest that leptin, IL-6 and suPAR are associated distinctively with various complications of both ageing and HIV-infection. Identifying major contributors to circulating leptin, IL-6, and suPAR levels could therefore improve our understanding of which processes leads to distinct age-related complications.

\section{Methods}

Microdialysis results from 18 subjects of the study have been published as part of a microdialysis methodological study [49].

\section{Ethics}

The local ethics committee of the Capital Region of Denmark (H-4-2010-045), and the Danish Data protection agency (2010-41-4952) approved the study. We carried out the study according to the declaration of Helsinki. Written informed consent was obtained from all participants.

\section{Subjects}

We included 60 HIV-infected patients from the Department of Infectious Diseases, Copenhagen University Hospital, Hvidovre, Denmark, and 16 healthy men (Controls) from November 2010 through October 2012. Controls were recruited by advertisement at Hvidovre Hospital. Inclusion criteria were: male; age older than 18 years; White ethnicity; no current Hepatitis B (HBV) and/or C (HCV) infection; no current immunomodulating treatment or disease; no current lipid-lowering, anti-diabetic or endocrinologic treatment; no intravenous drug use. Moreover, the HIVinfected patients also had to fulfil: at least 12 month on cART; CD4+ cell counts $\geq 200$ cells $/ \mu L ;$ HIV-RNA $<400$ copies/mL. Controls were tested negative for HIV, HBV, and $\mathrm{HCV}$ before inclusion, and it was ensured that mean age was similar in patients and Controls.

\section{Study protocol}

Blood samples were collected in the morning after an overnight fast of eight hours. All subjects completed a self-administered questionnaire including: tobacco use; AT distribution; and physical activity. Anthropometry was performed as in Andersen et al. [50], and included: body weight, height, waist and hip circumference. Blood pressure was measured after minimum one hour of rest. All participants were told to refrain from vigorous exercise the day before examination, physical activity on the examination day, and the use of any pain killers the week before the examination.

Whole body composition was evaluated by Dual energy X-ray absorptiometry (DXA) scan (Norland XR-36, Gammatec A/S, Værløse, Denmark). An experienced 
radiologist evaluated abdominal SAT and VAT mass by single slice computed tomography (CT) scan (Somatom Sensation 10, Siemens) at the upper limit of L4 as previously described in Hansen et al. [51].

Lipodystrophy was determined by physical examination by a trained examiner. Fat atrophy or hypertrophy in the face, retroauricular region, dorsocervical region, upper arms, thighs, buttocks, and abdominal subcutaneous and visceral regions was assessed. Patients were characterised as lipodystrophic (Lipo) if at least one sign of atrophy and/or hypertrophy was present, as in Andersen et al. [50]. Patients were not characterised as lipodystrophic (Non-Lipo) if no other signs of lipodystrophy than abdominal obesity were present. Patients were characterised as atrophic (Atrophy) if only signs of lipoatrophy were present; hypertrophic (Hyper) if only signs of lipohypertrophy were present, and Mixed type (Mix) if both lipoatrophy and lipohypertrophy was present.

\section{Body composition measures}

Total and regional lean and fat mass was derived from DXA scans. Lean mass was used as a surrogate marker for muscle mass, since lean mass is primarily constituted of skeletal muscle, especially appendicular lean mass [52]. Fat and lean mass measures were constructed as previously described in Kelly et al. [53]: Fat mass index (FMI) was evaluated as: total fat mass $/$ height ${ }^{2}\left(\mathrm{~kg} / \mathrm{m}^{2}\right)$. Total lean mass index $(t \mathrm{LMI})$ as: total lean mass $/$ height ${ }^{2}\left(\mathrm{~kg} / \mathrm{m}^{2}\right)$. Leg lean mass index (lLMI) as: lean mass of legs $/$ height $^{2}\left(\mathrm{~kg} / \mathrm{m}^{2}\right)$. Central SAT as: SAT area $\left(\mathrm{cm}^{2}\right)$. Central VAT as: VAT area $\left(\mathrm{cm}^{2}\right)$. Metabolic syndrome was assigned according to the 2009 metabolic syndrome consensus definition [54]. We used $\geq 94 \mathrm{~cm}$ as waist circumference cut-off as recommended for high risk populations.

\section{Blood sample measurements}

CD4+ and CD8+ T-cell numbers, HIV RNA, and glucose were measured as part of the routine patient management. Insulin was measured in plasma by the Immulite 2000 Systems (Siemens ${ }^{\oplus}, \mathrm{NY}$, USA). Plasma suPAR was measured with the suPARnostic ${ }^{\circ}$ ELISA (ViroGates A/S, Birkerød, Denmark) with a mean coefficient of variation (CV) of $4 \%$. Plasma IL-6 was measured with Quantikine HS-IL-6 ELISA (R\&D Systems, Minneapolis, USA) with a mean $\mathrm{CV}$ of $8 \%$. Plasma leptin was measured with Quantikine $^{\curvearrowleft}$ Leptin ELISA (R\&D Systems, Minneapolis, USA) with a mean CV of $3 \%$. Insulin resistance was calculated using the homeostatic model assessment of insulin resistance (HOMA-IR) as described by Matthews et al. [55].

\section{Statistics}

We assessed the effect of lipodystrophy on biomarker levels by comparing biomarker levels in lipodystrophy subgroups and Non-Lipo, and of HIV/cART by comparing biomarker levels in Non-Lipo and Controls. Differences in baseline characteristics between groups were analysed by one-way Anova models. If residuals were not normally distributed, we used Kruskal-Wallis tests. Group differences in smoking, HIV, previous thymidine nucleoside reverse transcriptase inhibitor (tNRTI) exposure, RNA >20 copies/mL, and metabolic syndrome were analysed by $\chi^{2}$ or Fisher's exact test if less than five individuals were expected in a group.

The association of biomarkers with fat and lean mass was analysed by linear regression analyses and tested for group interactions with biomarker level as outcome. The association between fat and lean mass was analysed in a similar manner with lean mass as outcome. The association of biomarkers with age, group, smoking, fat and lean mass, was analysed in univariate analyses and multiple regression analyses adjusted for biological relevant covariates with biomarker level as outcome.

We investigated goodness of fit of the linear regression models for normal distribution of residuals and homogeneity of variance. If residuals were not normal, biomarker levels were transformed using $\log _{2}(\mathrm{x})$ to obtain normal distributed residuals. Results from regression analyses were therefore back-transformed using $1-2^{\beta}$, and shown as percent estimates per unit increase. $P$-values less than 0.05 were considered statistically significant. The statistics program"Statistical Analysis Systems" (SAS, version 9.3; SAS Institute, Cary, NC, USA) was applied for analyses. Graphs were made using Graph Pad Prism (GraphPad Software Inc., San Diego, CA, USA) and the statistical software R (version 2.15.2, $\mathrm{R}$ foundation).

\section{Abbreviations}

AT: Adipose tissue; CART: combination antiretroviral therapy; C/EBP: CCAAT-enhancer-binding proteins; $C$ : Computed tomography; DXA: Dual energy X-ray absorptiometry; FMI: Fat mass index; IL-6: Interleukin 6; HDL: High density lipoprotein; HOMA-IR: Homeostasis model assessment of insulin resistance; LDL: Low density lipoprotein; /LMI: Leg lean mass index; tLMI: Total lean mass index; SAT: Subcutaneous adipose tissue; Sp-1: Specificity protein 1; suPAR: Soluble urokinase plasminogen activator receptor; tNRTI: Thymidine nucleoside reverse transcriptase inhibitors; VAT: Visceral adipose tissue; VLDL: Very low density lipoprotein.

\section{Competing interest}

ViroGates A/S donated the suPARnostic ${ }^{\oplus}$ kits for suPAR measurements, but did not have any influence on the design of the study. Jesper Eugen-Olsen and Ove Andersen are inventors of the patent on suPAR and disease risk. Copenhagen University Hospital, Hvidovre owns the patent, which is licensed to ViroGates AVS. Jesper Eugen-Olsen is founder, board member and shareholder in ViroGates A/S. Anne Langkilde, Janne Petersen, Jens Henrik Henriksen, Frank Krieger Jensen, and Jan Gerstoft declare no conflicts of interest.

\section{Authors' contributions}

AL participated in the design of the study; the recruitment and inclusion of patients; the statistical analyses; carried out the laboratory measurements; and drafted the study. JP participated in the design of the study and 
conceived the statistical analyses. $\mathrm{JHH}$ participated in the coordination of the DXA scans and the drafting of the manuscript. FKJ evaluated the CT scans. JG participated in patient recruitment. JEO participated in the study design and drafting of the study. OA participated in the design of the study, recruitment and inclusion of patients, and drafting the study. All authors read and approved the final manuscript.

\section{Acknowledgements}

We are very grateful to the individuals of this study for their participation. Moreover, we wish to thank the staff at Department of Infectious Diseases, Copenhagen University Hospital, Hvidovre, for assisting in patient-recruitment. We are very thankful to Michele Weber, Kristian Zobbe, and Henrik Hedegaard Klausen for technical support and assistance at examinations. Moreover we are very grateful for the financial support from Aase and Ejnar Danielsens Fond, and Arvid Nilssons Fond.

\section{Author details}

${ }^{1}$ Clinical Research Centre, Copenhagen University Hospital, Hvidovre, Kettegård Alle 30, DK-2650 Hvidovre, Denmark. ${ }^{2}$ Department of Clinical Physiology and Nuclear Medicine, Copenhagen University Hospital, Hvidovre, Kettegård Alle 30, DK-2650 Hvidovre, Denmark. . ${ }^{3}$ epartment of Radiology, Copenhagen University Hospital, Hvidovre, Kettegård Alle 30, DK-2650 Hvidovre, Denmark. ${ }^{4}$ Department of Infectious Diseases, Copenhagen University Hospital, Rigshospitalet, Blegdamsvej 9, DK-2100 København Ø, Denmark. ${ }^{5}$ Department of Infectious Diseases, Copenhagen University Hospital, Hvidovre, Kettegård Alle 30, DK-2650 Hvidovre, Denmark.

\section{Received: 9 March 2015 Accepted: 24 July 2015}

\section{Published online: 04 August 2015}

\section{References}

1. Lee JK, Bettencourt R, Brenner D, Le T-A, Barrett-Connor E, Loomba R. Association between serum interleukin- 6 concentrations and mortality in older adults: the rancho Bernardo study. Herder C, redaktør. PLoS One. 2012;7(4):e34218

2. Eugen-Olsen J, Andersen O, Linneberg A, Ladelund S, Hansen TW, Langkilde A, et al. Circulating soluble urokinase plasminogen activator receptor predicts cancer, cardiovascular disease, diabetes and mortality in the general population. J Intern Med. 2010;268(3):296-308.

3. Visser $M$, Pahor $M$, Taaffe DR, Goodpaster BH, Simonsick EM, Newman $A B$, et al. Relationship of interleukin-6 and tumor necrosis factor-alpha with muscle mass and muscle strength in elderly men and women: the Health ABC Study. J Gerontol A Biol Sci Med Sci. 2002;57(5):M326-32.

4. Ling PR, Schwartz JH, Bistrian BR. Mechanisms of host wasting induced by administration of cytokines in rats. Am J Physiol. 1997;272(3 Pt 1):E333-9.

5. Hotamisligil GS, Peraldi P, Budavari A, Ellis R, White MF, Spiegelman BM. IRS-1-mediated inhibition of insulin receptor tyrosine kinase activity in TNF-alpha- and obesity-induced insulin resistance. Science. 1996;271(5249):665-8

6. Ridker PM, Danielson E, Fonseca FAH, Genest J, Gotto Jr AM, Kastelein JJP, et al. Rosuvastatin to prevent vascular events in men and women with elevated C-reactive protein. N Engl J Med. 2008;359(21):2195-207.

7. Galli L, Salpietro S, Pellicciotta G, Galliani A, Piatti P, Hasson H, et al. Risk of type 2 diabetes among HIV-infected and healthy subjects in Italy. Eur J Epidemiol. 2012;27(8):657-65.

8. Neuhaus J, Jacobs DR, Baker JV, Calmy A, Duprez D, La RA, et al. Markers of inflammation, coagulation, and renal function are elevated in adults with HIV infection. J Infect Dis. 2010;201(12):1788-95.

9. Guaraldi G, Orlando G, Zona S, Menozzi M, Carli F, Garlassi E, et al. Premature age-related comorbidities among HIV-infected persons compared with the general population. Clin Infect Dis. 2011;53(11):1120-6.

10. Buehring B, Kirchner E, Sun Z, Calabrese L. The frequency of low muscle mass and its overlap with low bone mineral density and lipodystrophy in individuals with HIV_a pilot study using DXA total body composition analysis. J Clin Densitom Off J Int Soc Clin Densitom. 2012;15(2):224-32.

11. Haren MT, Malmstrom TK, Miller DK, Patrick P, Perry 3rd HM, Herning MM, et al. Higher C-reactive protein and soluble tumor necrosis factor receptor levels are associated with poor physical function and disability: a cross-sectional analysis of a cohort of late middle-aged African Americans. J Gerontol A Biol Sci Med Sci. 2010;65(3):274-81.

12. Nguyen A, Calmy A, Schiffer V, Bernasconi E, Battegay M, Opravil M, et al. Lipodystrophy and weight changes: data from the Swiss HIV Cohort Study, 2000-2006. HIV Med. 2008;9(3):142-50.

13. Andany N, Raboud JM, Walmsley S, Diong C, Rourke SB, Rueda S, et al. Ethnicity and gender differences in lipodystrophy of HIV-positive individuals taking antiretroviral therapy in Ontario, Canada. HIV Clin Trials. 2011;12(2):89-103.

14. Carr A, Emery S, Law M, Puls R, Lundgren JD, Powderly WG. An objective case definition of lipodystrophy in HIV-infected adults: a case-control study. Lancet. 2003;361(9359):726-35.

15. Nolan D, Hammond E, James I, McKinnon E, Mallal S. Contribution of nucleoside-analogue reverse transcriptase inhibitor therapy to lipoatrophy from the population to the cellular level. Antivir Ther. 2003;8(6):617-26.

16. Giralt $M$, Domingo $P$, Guallar JP, Rodriguez de la Concepcion ML, Alegre $M$, Domingo JC, et al. HIV-1 infection alters gene expression in adipose tissue, which contributes to HIV- 1/HAART-associated lipodystrophy. AntivirTher. 2006;11(6):729-40.

17. Sevastianova K, Sutinen J, Kannisto K, Hamsten A, Ristola M, Yki-Jarvinen H. Adipose tissue inflammation and liver fat in patients with highly active antiretroviral therapy-associated lipodystrophy. Am J Physiol Endocrinol Metab. 2008;295(1):E85-91.

18. Lihn AS, Richelsen B, Pedersen SB, Haugaard SB, Rathje GS, Madsbad S, et al. Increased expression of TNF-alpha, IL-6, and IL-8 in HALS: implications for reduced adiponectin expression and plasma levels. Am J Physiol Endocrinol Metab. 2003;285(5):E1072-80.

19. Schautz B, Later W, Heller M, Peters A, Müller MJ, Bosy-Westphal A. Impact of age on leptin and adiponectin independent of adiposity. Br J Nutr. 2012;108(2):363-70.

20. Moon H-S, Dalamaga M, Kim S-Y, Polyzos SA, Hamnvik O-P, Magkos F, et al. Leptin's Role in Lipodystrophic and Nonlipodystrophic Insulin-Resistant and Diabetic Individuals. Endocr Rev. 2013;34(3):377-12.

21. Nagy GS, Tsiodras S, Martin LD, Avihingsanon A, Gavrila A, Hsu WC, et al. Human immunodeficiency virus type 1-related lipoatrophy and lipohypertrophy are associated with serum concentrations of leptin. Clin Infect Dis. 2003;36(6):795-802.

22. Mulligan K, Khatami H, Schwarz J-M, Sakkas GK, DePaoli AM, Tai WW, et al. The effects of recombinant human leptin on visceral fat, dyslipidemia, and insulin resistance in patients with human immunodeficiency virus-associated lipoatrophy and hypoleptinemia. J Clin Endocrinol Metab. 2009:94(4):1137-44.

23. Sekhar RV, Jahoor F, lyer D, Guthikonda A, Paranilam J, Elhaj F, et al. Leptin replacement therapy does not improve the abnormal lipid kinetics of hypoleptinemic patients with HIV-associated lipodystrophy syndrome. Metabolism. 2012;61(10):1395-403.

24. Bonetto A, Aydogdu T, Jin X, Zhang Z, Zhan R, Puzis L, et al. JAK STAT3 pathway inhibition blocks skeletal muscle wasting downstream of IL-6 and in experimental cancer cachexia. Am J Physiol Endocrinol Metab. 2012:303(3):E410-21.

25. Pedersen BK, Febbraio MA. Muscles, exercise and obesity: skeletal muscle as a secretory organ. Nat Rev Endocrinol. 2012;8(8):457-65.

26. Cohen S, Nathan JA, Goldberg AL. Muscle wasting in disease: molecular mechanisms and promising therapies. Nat Rev Drug Discov. 2015;14(1):58-74.

27. Andersen O, Eugen-Olsen J, Kofoed K, Iversen J, Haugaard SB. Soluble urokinase plasminogen activator receptor is a marker of dysmetabolism in HIV-infected patients receiving highly active antiretroviral therapy. J Med Virol. 2008;80(2):209-16.

28. Thuno M, Macho B, Eugen-Olsen J. suPAR: the molecular crystal ball. Dis Markers. 2009;27(3):157-72.

29. Langkilde A, Petersen J, Klausen HH, Henriksen JH, Eugen-Olsen J, Andersen O. Inflammation in HIV-infected patients: impact of HIV, lifestyle, body composition, and demography - a cross sectional cohort study. PLoS One 2012;7(12):e51698.

30. Lyngbæk S, Sehestedt T, Marott JL, Hansen TW, Olsen MH, Andersen O, et al. CRP and suPAR are differently related to anthropometry and subclinical organ damage. Int J Cardiol. 2012;167(3):781-5.

31. Kohara K, Ochi M, Tabara $Y$, Nagai T, Igase M, Miki T. Leptin in sarcopenic visceral obesity: possible link between adipocytes and myocytes. PLoS One. 2011;6(9):e24633. 
32. Lee BT, Ahmed FA, Hamm LL, Teran FJ, Chen C-S, Liu Y, et al. Association of C-reactive protein, tumor necrosis factor-alpha, and interleukin-6 with chronic kidney disease. BMC Nephrol. 2015;16:77.

33. Wrann $C D$, Rosen ED. New insights into adipocyte-specific leptin gene expression. Adipocyte. 2012;1(3):168-72.

34. Isshiki H, Akira S, Tanabe O, Nakajima T, Shimamoto T, Hirano T, et al. Constitutive and interleukin-1 (IL-1)-inducible factors interact with the IL-1-responsive element in the IL-6 gene. Mol Cell Biol. 1990;10(6):2757-64.

35. Kang SH, Brown DA, Kitajima I, Xu X, Heidenreich O, Gryaznov S, et al. Binding and functional effects of transcriptional factor Sp1 on the murine interleukin-6 promotor. J Biol Chem. 1996;271(13):7330-5.

36. Loffreda S, Yang SQ, Lin HZ, Karp CL, Brengman ML, Wang DJ, et al. Leptin regulates proinflammatory immune responses. FASEB J Off Publ Fed Am Soc Exp Biol. 1998;12(1):57-65.

37. Trujillo ME, Sullivan S, Harten I, Schneider SH, Greenberg AS, Fried SK. Interleukin-6 regulates human adipose tissue lipid metabolism and leptin production in vitro. J Clin Endocrinol Metab. 2004;89(11):5577-82.

38. Weyer C, Foley JE, Bogardus C, Tataranni PA, Pratley RE. Enlarged subcutaneous abdominal adipocyte size, but not obesity itself, predicts type II diabetes independent of insulin resistance. Diabetologia. 2000;43(12):1498-506.

39. Skurk T, Alberti-Huber C, Herder C, Hauner H. Relationship between adipocyte size and adipokine expression and secretion. J Clin Endocrinol Metab. 2007;92(3):1023-33.

40. Cinti S, Mitchell G, Barbatelli G, Murano I, Ceresi E, Faloia E, et al. Adipocyte death defines macrophage localization and function in adipose tissue of obese mice and humans. J Lipid Res. 2005;46(11):2347-55.

41. Couillard C, Mauriège $P$, Imbeault $P$, Prud'homme $D$, Nadeau A, Tremblay A, et al. Hyperleptinemia is more closely associated with adipose cell hypertrophy than with adipose tissue hyperplasia. Int J Obes Relat Metab Disord J Int Assoc Study Obes. 2000;24(6):782-8.

42. Mohamed-Ali V, Goodrick S, Rawesh A, Katz DR, Miles JM, Yudkin JS, et al. Subcutaneous adipose tissue releases interleukin-6, but not tumor necrosis factor-alpha, in vivo. J Clin Endocrinol Metab. 1997;82(12):4196-200.

43. Senn JJ, Klover PJ, Nowak IA, Mooney RA. Interleukin-6 induces cellular insulin resistance in hepatocytes. Diabetes. 2002;51(12):3391-9.

44. Bryer SC, Koh TJ. The urokinase-type plasminogen activator receptor is not required for skeletal muscle inflammation or regeneration. Am J Physiol Regul Integr Comp Physiol. 2007;293(3):R1152-8.

45. DiPasquale DM, Cheng M, Billich W, Huang SA, van Rooijen N, Hornberger TA, et al. Urokinase-type plasminogen activator and macrophages are required for skeletal muscle hypertrophy in mice. Am J Physiol Cell Physiol. 2007;293(4):C1278-85.

46. Sandri M, Barberi L, Bijlsma AY, Blaauw B, Dyar KA, Milan G, et al. Signalling pathways regulating muscle mass in ageing skeletal muscle. The role of the IGF1-Akt-mTOR-FoxO pathway. Biogerontology. 2013;14(3):303-23.

47. Lluís F, Roma J, Suelves M, Parra M, Aniorte G, Gallardo E, et al. Urokinase-dependent plasminogen activation is required for efficient skeletal muscle regeneration in vivo. Blood. 2001;97(6):1703-11.

48. Vigouroux C, Maachi M, Nguyen TH, Coussieu C, Gharakhanian S, Funahashi T, et al. Serum adipocytokines are related to lipodystrophy and metabolic disorders in HIV-infected men under antiretroviral therapy. AIDS. 2003;17(10):1503-11.

49. Langkilde A, Andersen $\mathrm{O}$, Henriksen JH, Langberg H, Petersen J, Eugen-Olsen J. Assessment of in situ adipose tissue inflammation by microdialysis. Clin Physiol Funct Imaging. 2014;35(2):110-9.

50. Andersen O, Haugaard SB, Andersen UB, Friis-Moller N, Storgaard H, Volund $A$, et al. Lipodystrophy in human immunodeficiency virus patients impairs insulin action and induces defects in beta-cell function. Metabolism. 2003;52(10):1343-53.

51. Hansen BR, Haugaard SB, Jensen FK, Jensen JEB, Andresen L, Iversen J, et al. Long-term high-physiological-dose growth hormone reduces intra-abdominal fat in HIV-infected patients with a neutral effect on glucose metabolism. HIV Med. 2010;11(4):266-75.

52. Heymsfield SB, Wang Z, Baumgartner RN, Ross R. Human body composition: advances in models and methods. Annu Rev Nutr. 1997;17:527-58.

53. Kelly TL, Wilson KE, Heymsfield SB. Dual Energy X-Ray Absorptiometry Body Composition Reference Values from NHANES. PLoS One. 2009;4(9):e7038.

54. Alberti KGMM, Eckel RH, Grundy SM, Zimmet PZ, Cleeman Jl, Donato KA, et al. Harmonizing the metabolic syndrome: a joint interim statement of the International Diabetes Federation Task Force on Epidemiology and
Prevention; National Heart, Lung, and Blood Institute; American Heart Association; World Heart Federation; International Atherosclerosis Society; and International Association for the Study of Obesity. Circulation. 2009;120(16):1640-5.

55. Matthews DR, Hosker JP, Rudenski AS, Naylor BA, Treacher DF, Turner RC. Homeostasis model assessment: insulin resistance and beta-cell function from fasting plasma glucose and insulin concentrations in man. Diabetologia. 1985;28(7):412-9.

\section{Submit your next manuscript to BioMed Central and take full advantage of:}

- Convenient online submission

- Thorough peer review

- No space constraints or color figure charges

- Immediate publication on acceptance

- Inclusion in PubMed, CAS, Scopus and Google Scholar

- Research which is freely available for redistribution 